\title{
Compositional Verification of Reactive Systems Specified by Graph Transformation ${ }^{\star}$
}

\author{
Reiko Heckel ${ }^{1}$ \\ Dipartimento di Informatika, Università degli Studi di Pisa, \\ Corso Italia, 40, I - 56125 Pisa, Italia, e-mail: reiko@di.unipi.it
}

\begin{abstract}
A loose semantics for graph transformation rules which has been developed recently is used in this paper for the compositional verification of specifications. The main conceptual tool here is the notion of view, that is, an incomplete specification describing only a certain aspect of the overall system. A view anticipates the (potential) behavior of the complete system by its loose semantics. This ensures that properties of the view are inherited by the complete system.

Based on this result one may verify temporal properties by decomposing a specification into several views, analyzing them separately, and deriving the desired property from properties shown for the views.
\end{abstract}

\section{Introduction}

One of the most challenging problems in specifying reactive systems is the complexity of the development and verification of large specifications. An important approach to solve this problem is the separation of different aspects or views. They may be used to split a team of developers into subgroups specifying only that aspect of a system which is later responsible for a certain task or is seen and used by a certain type of user. In the software engineering field, this vieworiented approach is known by the notion of viewpoints (cf. e.g., [FKN ${ }^{+}$92]).

Reactive systems in the sense of [MP92] are distinguished by the complex interaction between the system and its environment (as opposed to classical programs which basically transform input to output). They include concurrent, object-oriented, or distributed systems like operating systems, process control systems, seat reservation systems, etc., typically consisting of several components which can be seen as reactive systems themselves.

Probably, the most natural representation of such a system is a graph, where nodes represent components (objects, processes, ...) and edges communication links. If the system's topology is dynamic (since e.g., components are created and deleted) some sort of graph transformation has to be performed. Among the

\footnotetext{
* Research supported by the TMR network GETGRATS and the ESPRIT BRWG APPLIGRAPH.
} 
various possibilities of specifying the transformation of graphs, I prefer a rulebased approach which exploits the visual character of the graph representation for describing transformations. In particular, the "algebraic, Double Pushout (DPO) approach" is applied [EPS73,CMR ${ }^{+97]}$, which owes its name to the basic construction of a direct derivation step: This is modeled indeed by two gluing diagrams (i.e., pushouts) in the category of graphs and total graph morphisms.

In [EHTE97] a specification technique based on graph transformations has been presented which supports a view-based development approach. Since each view specifies only a part of the operations, it may be that a view operation is executed in parallel with operations of other views. Thus, a view operation specifies only what at least has to happen on a system's state, but it allows also additional effects which may be caused by the environment. In this sense, the semantics of a view is a loose one, in contrast to the semantics of the complete specification. A loose operational semantics for graph transformation rules, called graph transitions as opposed to direct derivations, has been developed in [HEWC97b] and used in [EHTE97] as the semantics of views.

Here, this loose semantics is exploited for compositional reasoning about reactive systems. The hope is that a meaningful decomposition of a specification into views reduces the complexity of the verification and, which seems equally important, that it allows to concentrate on the verification of the most important (e.g., safety-critical) aspects.

The general idea of compositionality through loose semantics can be traced back to several sources. In the area of algebraic specification a class of algebras forms the loose semantics of an incomplete specification, as opposed to a single (initial) algebra associated to a complete specification. Extending a specification (e.g., by adding equations) reduces its class of models [EM85]. Similar to the present approach is [MP92], where a loose interpretation of assignments in a shared variable languages (allowing for unspecified change of values) is used for compositional proofs of temporal properties.

The approach here is to verify temporal properties of a big specification by decomposing it into views and analyzing them separately. Then, the properties of the big specification are derived from properties of the views. The verification of properties of views is not addressed in this paper. The main technical result shows the correctness of this compositional verification approach, that is, temporal properties of the views remain valid in the complete specification. The technique is applied to a small case study specifying an algorithm for mutual exclusion and deadlock detection in a distributed system with dynamic topology.

\section{Specifying a Distributed Algorithm with Graph Transformation Rules}

The following specification of a distributed mutual exclusion (MUTEX) algorithm shall illustrate the expressive power of rule-based graph transformation and serves as running example throughout the paper. The ideas are standard in distributed computing and have been communicated to me through [Sch94]. 
Along with this specification the basic notions of the algebraic DPO approach to graph transformation [EPS73,CMR ${ }^{+97]}$ are introduced.

Distributed MUTEX algorithms are used in (distributed) operating systems and data base systems. Their task is to ensure that a resource is never used by more than one process at a time. However, each request of a process for a resource must eventually be granted. Here the main problem is to prevent processes from running into a deadlock.

Graph transformation rules are used for specifying a MUTEX algorithm with deadlock detection for a variable number of processes and resources. The specification is presented in three views, the system view (SYS) specifying the creation and deletion of processes and resources, the token ring view (TR) ensuring mutual exclusion, and the distributed deadlock detection view (DDD) detecting and announcing deadlocks. All views share a common graph signature specifying the structure of system states. They use disjoint sets of graph transformation rules for describing the evolution of the system.

A graph signature is an algebraic signature $G S=\langle S, O P\rangle$ having only unary operation symbols $o p: s \rightarrow s^{\prime}{ }^{1}$ GS-Algebras and -homomorphisms are called graphs and graph morphisms, respectively. ${ }^{2}$ The category of graphs and graph morphisms for a signature $G S$ is denoted by $\mathcal{G} r(G S)$. In the MUTEX specification type graphs (in the sense of e.g., [HCEL96]) are used as a more intuitive, graphical notation for signatures.
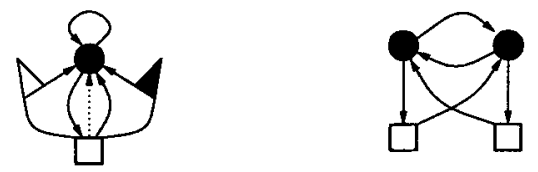

Fig. 1. Type graph of MUTEX (left) and deadlock state (right)

Example 1. The type graph of the MUTEX specification is shown in the left of Figure 1. It may be read like an entity/relationship schema specifying the node and edge types which may occur in graphs modeling system states. Processes are drawn as black nodes and resources as light boxes. An edge from a process to a resource models a request. A solid edge in the opposite direction shows that the resource is currently held_by the process. A dashed edge from a resource to a process asks the process to release the resource.

The token ring is a cyclic list of processes, where an edge between two processes points to the next process. For each resource there is a token, represented

${ }^{1}$ Some basic notions of universal algebra are assumed, like signature Sig, Sig-algebra $A$ and Sig-homomorphism $h: A \rightarrow B$, subsignature Sig' $\subseteq$ Sig, and Sig'-reduct $(A)_{S i g^{\prime}}$ and $(h)_{S i g^{\prime}}$, see e.g., [EM85].

2 Graphs of this kind have been mainly used in the algebraic single-pushout approach under the name of graph structures [Löw93]. They include, beside ordinary graphs, also more general kinds of structures like hyper-graphs or higher-order graphs. 
by an edge with a white flag, which is passed from process to process along the ring. If a process wants to use a resource, it waits for the corresponding token. Mutual exclusion is ensured because there is only one token for each resource in the system. The distributed deadlock detection uses blocked messages in order to detect cyclic dependencies. They are represented by edges with a black flag from a resource to a process.

The graph signature $G S_{M U T E X}=\langle S, O P\rangle$ has as sorts the nodes and edges of the type graph, i.e., the node sorts $P$ and $R$ and the edge sorts request, held_by, release, next,token, and blocked. The operations of the signature are the obvious source and target assignments from edge sorts to node sorts, like source : request $\rightarrow P$ and target : request $\rightarrow R$. A graph over this signature is shown in the right of Figure 1. It represents a system state with two processes and two resources, where each process requests one resource while holding the other one, i.e., both processes are deadlocked.

Graph transformation rules (or productions) according to the DPO approach are specified by spans of injective graph morphisms $s p=(L \stackrel{l}{\longleftarrow} K \stackrel{r}{\longrightarrow} R)$, called production spans. The left-hand side $L$ and the right-hand side $R$ represent pre- and postconditions, i.e., items which have to be present before and after the application. The interface graph $K$ specifies the objects which are required and preserved, that is, the "intersection" of $L$ and $R$.

Example 2. The rules of the MUTEX specification are given in Figure 2. In the upper part, the SYS-rules are shown modeling the operations new and kill $(p)$ for inserting and removing processes to and from the ring, and the operations mount and unmount $(r)$ for creating a resource with a token, and deleting it. The interface graph $K$ is omitted. It can be reconstructed from the graphical layout of productions: Items that appear in the same position in the left- and right-hand side are usually preserved and belong to the interface.

The complete representation of the production new is given by the top span of the left diagram in Figure 3 which shows an application modeling the insertion of a new process in the ring. Given the match $m: L \rightarrow G$ which maps the processes 1 and 2 to the process in $G$ and the edge in between to the loop, the application consists of two steps: The objects of $G$ matched by $L-l(K)$ are removed which leads to the graph $D$ without loop. Then, the objects of $R-r(K)$ are added to $D$ leading to the derived graph $H$. Notice that the application deletes and creates exactly what is specified by the production: There is an implicit frame condition ensuring that everything that is not rewritten explicitly by the production is left unchanged.

The gluing of $L$ and $D$ over $K$ is again the given graph $G$, i.e., the left-hand square (1) of $\boldsymbol{d}$ forms a so-called pushout complement. Only in this case the application is permitted. Similarly, the derived graph $H$ is the gluing of $D$ and $R$ over $K$, which creates the right-hand side pushout square (2). The resulting double-pushout (DPO) diagram $d$ represents the transformation of $G$ into $H$, denoted $G \stackrel{d}{\Longrightarrow} H$. Since this diagram is symmetric, it can also be read from 

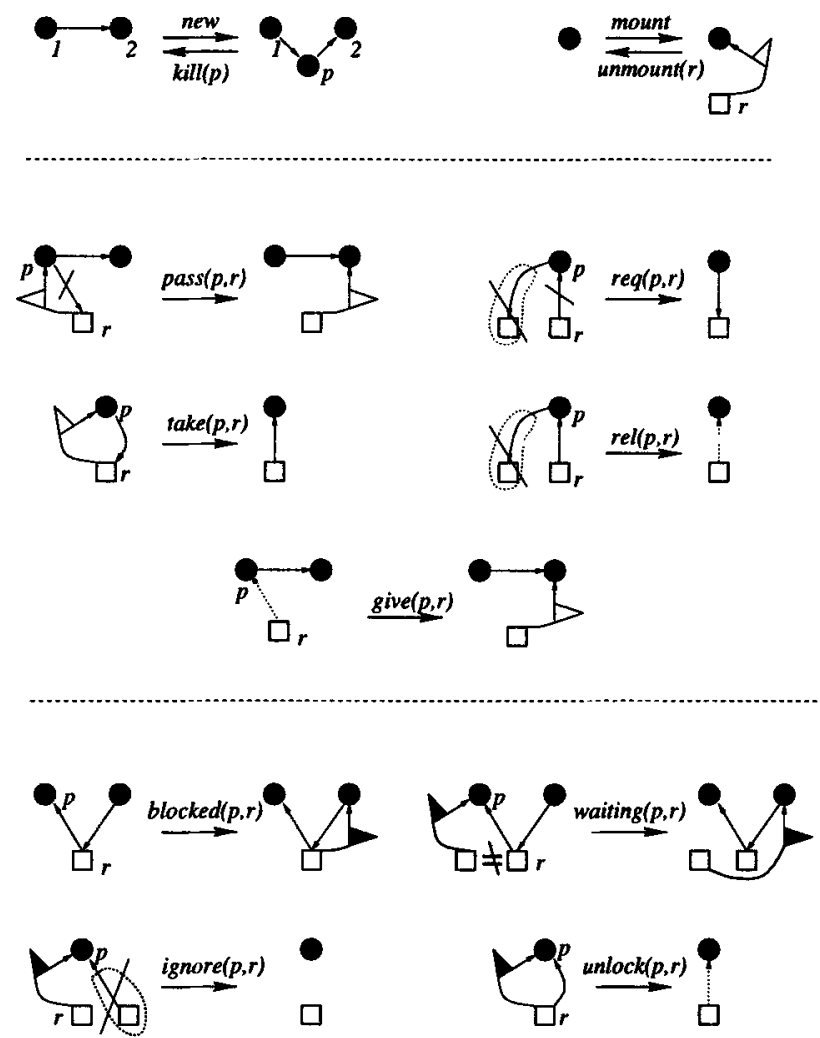

Fig. 2. The MUTEX-productions of the SYS-, TR- and DDD-view (from top to bottom).
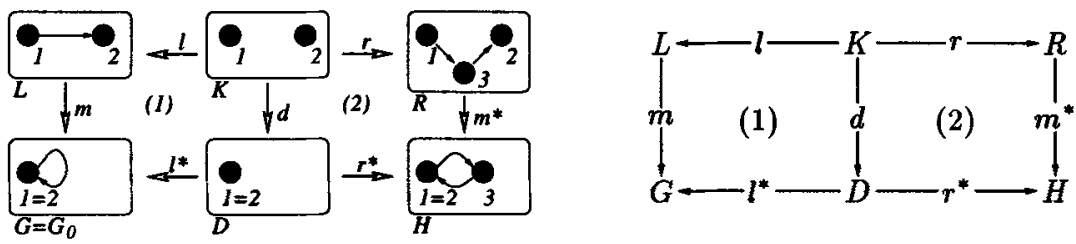

Fig. 3. A sample direct DPO derivation using new (left), and a generic double-pushout (resp., double-pullback) diagram $d$ (right). 
right to left, as inverse transformation. The corresponding inverse production span is $s p^{-1}=(R \stackrel{r}{\longleftarrow} K \stackrel{l}{\longrightarrow} L)$.

The existence of the pushout complement (1), and hence of a direct derivation $^{3} G \stackrel{d}{\Longrightarrow} H$, is characterized by the gluing conditions: The dangling condition ensures that the structure $D$ obtained by removing from $G$ all objects to be deleted is indeed a graph, that is, no edges are left "dangling" without source or target node. The identification condition states that objects from the left-hand side may only be identified by the match if they also belong to the interface (and are thus preserved).

Example 3. The TR-rules are shown in the middle of Figure 2, where pass $(p, r)$ describes that a process having the token may pass it to the next process in the ring, provided that it does not have a request on the corresponding resource. This negative application condition is visualized by the crossed-out request edge from the process to the resource, see [HHT96] for a formalization of this concept. If a process wants to use a resource, it may generate a request. This is modeled by the rule $r e q(p, r)$, which is only applicable if the process does not have any requests yet, and if the particular resource is not used already by this process. If a process receives a token and there is a request for the resource, the process will chose the rule take $(p, r)$ replacing the token and the request by a held_by edge from the resource to the process. When it has finished its task, the process may release its resource and give the token to the next process using $\operatorname{rel}(p, r)$ and give $(p, r)$. This will happen only when there are no pending requests, which is modeled by a negative application condition at $\operatorname{rel}(p, r)$.

The algorithm for distributed deadlock detection specified by the DDD-rules in the lower part is a variant of [CMH83]: In a graph representing a state, a deadlock is represented as a cycle of request and held_by edges. In order to detect such cycles in a distributed way, a process holding a resource requested by another process will use the rule blocked $(p, r)$ to send a blocked-message to that process. If this process itself blocks another one, he will use the waiting $(p, r)$ rule to pass this message on. The inequation in this rule, which is also a negative application condition in the sense of [HHT96], ensures that resource $r$ is not the original one. If the process does not hold any resource, it deletes the message with ignore $(p, r)$.

Thanks to the mutual exclusion, each resource is held by only one processes. Hence, if the message arrives at a process who holds the original resource, this is the original sender of the message. The deadlock thus detected is broken by the unlock $(p, r)$ rule which replaces the held_by edge and the blocked message with a release edge, asking the TR-view to give the token to the next process.

\section{Temporal Specification of Graph Transition Systems}

Graph transition systems are labeled transition systems whose states are graphs. In [HEWC97a] they are defined for a graph grammar (i.e., a set of productions

\footnotetext{
3 The pushout (2) always exists since the category of graphs over GS is cocomplete.
} 
with a start graph) using coalgebraic techniques. Following [GR97] in this section graph transition systems are defined for a signature, independently of their generation by productions. Like in [HEWC97a] a temporal logic is developed for specifying graph transition systems axiomatically.

The common base for graph transition systems, temporal formulas, and graph transition specifications (in the next section) are graph transition signatures:

Definition 4 (graph transition signature). A graph transition signature $G T S i g=\langle G S, C, P, Q\rangle$ consists of a graph signature $G S$, a global context graph $C$ over $G S$, and two families of sets $P=\left(P_{w}\right)_{w \in S^{*}}$ and $Q=\left(Q_{w}\right)_{w \in S^{*}}$ of production names and state formula symbols. For $p \in P_{v}$ and $q \in Q_{w}$ I write $p: v$ and $q: w$.

Given an $S$-indexed family of sets $A=\left(A_{s}\right)_{s \in S}$, the set of transition expressions over $A$ is defined by $\mathcal{T} \mathcal{E}_{P}(A)=\left\{p(a) \mid p: w, a \in A_{w}\right\}$. Similarly, the set of state formulas over $A$ is defined by $\mathcal{S F}_{Q}(A)=\left\{q(a) \mid q: w, a \in A_{w}\right\}$.

Example 5. The graph transition signature of the MUTEX algorithm is GTSig MUTEX $=\left\langle G_{M U T E X}, C_{M U T E X}, P_{S Y S} \cup P_{T R} \cup P_{D D D}, Q_{M U T E X}\right\rangle$ where

- GS $S_{M U T E X}$ is the graph signature introduced in Example 1

$-C_{M U T E X}=\left\langle\left(C_{s}\right)_{s \in S},\left(o p^{C}\right)_{o p \in O P}\right\rangle$ is a "complete graph structure" of size $\mathbb{N}$, that is, $C_{s}=\mathbb{N}$ for all node sorts $s$ and $C_{s^{\prime}}=\mathbb{N} \times \mathbb{N} \times \mathbb{N}$ for all edge sorts $s^{\prime}$. The source and target mappings from node to edge sorts are given, respectively, by the first and third projection. Hence $C_{M U T E X}$ has for each node type all natural numbers as nodes, and for each edge type the set of natural numbers as edges between every pair of nodes.

- $P_{S Y S}=\{$ new, kill $: P$, mount, unmount $: R\}$, $P_{T R}=\{$ pass $: P R$, req $: P R$, take $: P R$, rel $: P R$, give $: P R\}$, and $P_{D D D}=\{$ blocked $: P R$, waiting $: P R$, ignore $: P R$, unlock $: P R\}$.

$-Q_{M U T E X}=\{$ no_request $: P R$, no_request $: P$, not_held_by $: R P, \ldots\}$

The global context graph $C$ provides a common name space for the states, formally represented as injective graph morphisms $g: G \rightarrow C$. The set of all states is denoted by $S_{C}$. Transitions are labeled by sets of transition expressions representing parallel transitions. For evaluating temporal formulas, a valuation $\mathcal{V}$ of state formulas over $C$ is assumed.

Definition 6 (graph transition system). A graph transition system over a signature $G T S i g=\langle G S, C, P, Q\rangle$ is a triple $G T S y s=\langle\mathcal{V}, \sim, \mathcal{R}\rangle$ where

- $\mathcal{V}: S \mathcal{F}_{Q}(C) \rightarrow \mathcal{P}\left(S_{C}\right)$ is a valuation assigning to each state formula $q(c)$ over $C$ a class $\mathcal{V}(q(c))$ of states (at which $q(c)$ is assumed to be true)

- $\sim \subseteq S \times \mathcal{P}_{f}\left(\mathcal{T} \mathcal{E}_{P}(C)\right) \times S$ is a labeled transition relation ${ }^{4}$, where $\langle g, L, h\rangle \in$ $\leadsto$ is denoted by $g \stackrel{L}{\sim} h$.

- $\mathcal{R}$ is a class of runs, i.e., infinite sequences $\rho: g_{0} g_{1} g_{2} \ldots$ of states over $C$ such that for all $i \in \mathbb{N}$ there exists a transition $g_{i} \stackrel{L_{i}}{\rightarrow} g_{i+1}$.

${ }^{4}$ For a set $A, \mathcal{P}_{f}(A)$ denotes the set of finite subsets of $A$. 
Graph transition systems can be specified axiomatically (via their runs) by temporal logic formulas. Assume in the following definitions a graph transition signature $G T S i g=\langle G S, C, P, Q\rangle$.

Definition 7 (temporal formulas). Given a family of sets of variables $X=$ $\left(X_{s}\right)_{s \in S}$, the syntax of temporal formulas over $X$ has the form

$$
\Phi::=q(x)|\neg \Phi| \Phi_{1} \wedge \Phi_{2}|\exists y . \Phi| \diamond \Phi
$$

where $q(x)$ is a state formula over $X$, and $y \in X$ is a variable. A temporal formula with variables is a pair $X: \Phi$ where $\Phi$ is a temporal formula over $X$. The set of all such formulas is $\mathcal{T} \mathcal{F}_{G T S i g}$.

Here, $\diamond$ is the usual sometime operator of temporal logic. The always operator is defined by: $\square \Phi$ iff $\neg \diamond \neg \Phi$. The usual definitions are applied for the propositional operators $T, F, \vee$, and $\Longrightarrow$, and for the universal quantification $\forall$.

Satisfaction of temporal formulas is defined for a temporal model $M=$ $\langle G T S y s$, ass $\rangle$ over GTSig consisting of a graph transition system GTSys = $\langle\mathcal{V}, \sim, \mathcal{R}\rangle$ over GTSig, and an assignment ass $: X \rightarrow C$.

Definition 8 (satisfaction). Let $M=\langle G T S y s, a s s\rangle$ be a temporal model. The satisfaction of temporal formulas $X: \Phi \in \mathcal{T} \mathcal{F}_{\text {GTSig }}$ by runs $\rho \in \mathcal{R}$ is defined inductively as follows: ${ }^{5}$

- $\rho \vDash_{M} q(x)$ iff $\rho(0) \in \mathcal{V}(q(a s s(x)))$

$-\rho \vDash_{M} \neg \Phi$ iff $\rho \nvdash_{M} \Phi$,

$-\rho \vDash_{M} \Phi_{1} \wedge \Phi_{2}$ iff $\rho \vDash_{M} \Phi_{1}$ and $\rho \models_{M} \Phi_{2}$,

$-\rho \vDash_{M} \exists y . \Phi$ iff there is an assignment $a s s^{\prime}$ s.t. $a s s^{\prime}(x)=a s s(x)$ for all variables $x \neq y$ and $\rho \vDash\left\langle G T S y s, a s s^{\prime}\right\rangle$

$-\rho \vDash_{M} \diamond \Phi$ iff there is $k \in \mathbb{N}$ such that $\rho_{k} \vDash_{M} \Phi$.

The model $M$ satisfies $X: \Phi$, written $\models_{M} X: \Phi$, if $\rho \models_{M} \Phi$ for all runs $\rho \in \mathcal{R}$. A transition system $G T S y s$ satisfies $X: \Phi$, written $\models_{G T S y s} X: \Phi$, if $\models_{M} X: \Phi$ for all models $M=\langle$ GTSys, ass $\rangle$.

Example 9. The temporal specification of the MUTEX algorithm consists of the safety property MUTEX-safe: $\square\left[\right.$ held_by $\left(r, p_{1}\right) \wedge$ held_by $\left.\left(r, p_{2}\right) \Longrightarrow p_{1}=p_{2}\right]$ which states that a resource must not be held by two different processes, and the liveness property MUTEX-live: $\square[$ requests $(p, r) \Longrightarrow \diamond$ held_by $(r, p)]$ saying that each request will eventually be served. The set of state formulas is extended by equations and edge predicates, like edge_sort : node_sort ${ }_{1}$ node_sort $_{2}$, with the obvious interpretations.

The MUTEX liveness property is decomposed according to the different tasks performed by the TR- and the DDD-view. The latter has to announce all permanent deadlocks by a release edge, that is, $D D D$-live reads:

$\square\left[\right.$ deadlocked $\left(p_{1}\right) \Longrightarrow \exists p_{2}, r . \operatorname{path}\left(p_{1}, p_{2}\right) \wedge \diamond\left[\operatorname{release}\left(r, p_{2}\right) \vee \neg\right.$ deadlocked $\left.\left.\left(p_{1}\right)\right]\right]$ $\overline{5} \rho(i)=g_{i}$ denotes the $i$ th state and $\rho_{i}=g_{i} g_{i+1} \ldots$ the $i$ th suffix of $\rho$. 
Here $\operatorname{path}\left(p_{1}, p_{2}\right)$ is true in a state if the processes $p_{1}$ and $p_{2}$ are connected by a path of request and held_by edges, and deadlocked $(p)$ is equivalent to path $(p, p)$. Notice that the release edge may point to any process in the deadlock cycle.

The liveness property of the TR-view is that each request will eventually be served, provided that deadlocks are always detected and the number of processes is bounded, i.e., TR-live : $\square$ bounded $(n) \wedge D D D$-live $\Longrightarrow M U T E X$-live. Here bounded $(n)$ is true in a state if the number of $P$ nodes does not exceed $n$.

\section{A Loose Semantics for Graph Productions}

A graph transition system (or rather its transition relation) can be specified constructively by a set of graph productions (plus a set of initial states and some additional constraints). One can ask if this specification is correct w.r.t. a certain temporal formula by checking the validity of this formula in the transition system. If the specification is incomplete like the DDD-view which describes only a particular aspect of the MUTEX algorithm, the closed behavior of this view given by the classical DPO interpretation is rather poor. Starting for example with the deadlock state in Figure 1 on the right, the DDD-productions could (at most) replace two held_by edges by release edges, and stop. Properties verified for this transition system are obviously not very interesting and of little use for the verification of the MUTEX algorithm.

One way out is, of course, to verify the DDD-view in the context of the SYSand TR-view, but this would contradict the aim of compositional verification. The solution I propose in this paper is a loose semantics of graph productions which anticipates the effects of applying them in a bigger context. So-called graph transitions will ensure that an application preserves, deletes, and adds at least as much as it is specified by the productions, but it permits also additional effects which may be caused, in the example, by concurrent application of the SYS- and TR-productions. Thus the implicit frame condition is dropped. Instead, explicit frame conditions are introduced which protect only particular parts of the graphs from unspecified changes.

Definition 10 (graph transition). A sequential graph transition is defined by replacing the double-pushout diagram of direct derivations with a double-pullback (DPB) diagram, that is, a diagram $d$ like in the right of Figure 3 where (1) and (2) are pullbacks. This diagram represents a transition from $G$ to $H$ with production span $s p=(L \longleftarrow K \longrightarrow R)$ and bottom span $t=(G \longleftarrow D \longrightarrow H)$, denoted by $G \stackrel{d}{\sim} H$.

The parallel production span $\mathcal{P} a r(S p)$ of a finite set $S p$ of production spans $s p=\left(L_{s p} \longleftarrow K_{s p} \longrightarrow R_{s p}\right)$ is constructed as component-wise disjoint union (coproduct)

$$
\operatorname{Par}(S p)=\sum_{s p \in S p} L_{s p} \stackrel{l}{\longleftarrow} \sum_{s p \in S p} K_{s p} \stackrel{r}{\longrightarrow} \sum_{s p \in S p} R_{s p}
$$

A graph transition using a parallel production span is called parallel graph transition. 
Each pushout in $\mathcal{G} r(G S)$ where two opposite morphisms are injective is also a pullback. Hence, graph transitions generalize direct derivations defined by double-pushouts. From an operational point of view a span $G \stackrel{\stackrel{*}{*}^{*}}{\stackrel{r^{*}}{\longrightarrow}} H$ represents a transition where $G-l^{*}(D)$ is deleted, $l^{*}(D) \subseteq G$ is preserved as $r^{*}(D) \subseteq H$, and $H-r^{*}(D)$ is newly created. Then, referring to the right diagram of Figure 3:

- Commutativity of (1) and (2) ensures that the image of $K$ in $G$ is preserved in $D$ and $H$, i.e., $m(l(K)) \subseteq l^{*}(D)$ and $m^{*}(r(K)) \subseteq r^{*}(D)$.

- Pullback property of (1) ensures that at least every image of $L-l(K)$ in $G$ is deleted, i.e., $m(L-l(K)) \cap l^{*}(D)=\emptyset$.

- Pullback property of (2) ensures that at least every image of $R-r(K)$ in $H$ is newly created, i.e., $m^{*}(R-r(K)) \cap r^{*}(D)=\emptyset$.

Example 11. A sample graph transition is shown in the left of Figure 4. It applies the TR-production $\operatorname{take}(p, r)$ replacing a request and the corresponding token by a held_by edge. Meanwhile, another process is inserted in the ring, which is not specified by the production but permitted by the loose semantics. In fact, none of the two squares is a pushout: The given graph $G$ adds to the gluing of $L$ and $D$ a next loop which is "spontaneously deleted", and in the derived graph $H$, an additional process with two next edges has been "spontaneously created". This effect could be obtained by applying production new in parallel.
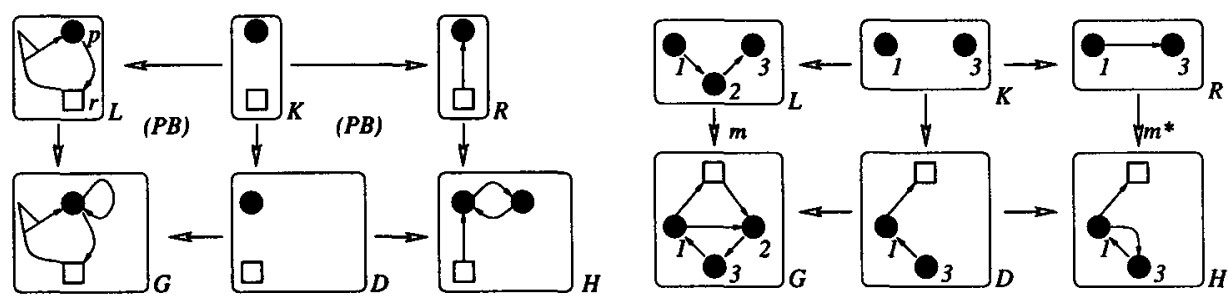

Fig. 4. A sample graph transition (left) and a graph transition not satisfying the dangling condition (right).

It is worth stressing that graph transitions may not only have additional effects but are also more likely to exist than DPO derivations. In general, the match $m$ of a DPB diagram may satisfy neither the identification nor the dangling condition of the corresponding production, and so does the comatch $m^{*}$. Consider for example the transition in the right of Figure 4, using the SYSproduction $k i l l(p)$, where the match does not satisfy the dangling condition: Process 2 is removed from the ring while still holding the resource. Here deleting the dangling held_by edge is an unspecified effect. Symmetrically, it would be possible to attach edges to newly created processes (for example in the inverse transition). 
Motivated by these observations safe transitions are introduced:

Definition 12 (safe transitions). A graph transition $G \stackrel{d}{\sim} H$ like in the right of Figure 3 is safe if $m$ satisfies the gluing conditions w.r.t. $s p=(L \longleftarrow K \longrightarrow$ $R$ ), and $m^{*}$ satisfies the gluing conditions w.r.t. $s p^{-1}=(R \longleftarrow K \longrightarrow L)$.

In the following all transitions are assumed to be safe.

Hence, a safe graph transition exists if and only if there is also a direct DPO derivation with the same match.

In order to ensure that, e.g., processes and resources are only created and deleted by SYS-productions, one may declare certain sorts as input and/or output sorts. Only elements of such sorts may be spontaneously created and deleted. Sorts that are neither input nor output are called private since they are protected from the influence of the environment.

Definition 13 (explicit frame condition). An explicit frame condition over $G S$ is a pair of sets $F C=\langle I S, O S\rangle$ of input and output sorts $I S, O S \subseteq S$. Denote by $\overline{I S}(\overline{O S})$ the biggest subsignature of $G S$ not containing input (output) sorts. Then, a graph transition $G \stackrel{d}{\sim} H$ respects $I S(O S)$ if the reduct of the left-hand side (right-hand side) pullback square of $d$ to $\overline{I S}(\overline{O S})$ is a pushout. ${ }^{6}$

If all sorts are private (that is, $F C=\langle\emptyset, \emptyset\rangle$ ), transitions are reduced to direct derivations. In this way, the classical DPO interpretation of productions becomes a special case of the loose one.

As constructive specification of a graph transition system, production definitions interpret production names by spans with application conditions. Together with frame conditions, initial graphs, and fairness conditions for runs, this forms a graph transition specification.

Definition 14 (graph transition specification). A graph transition specification over a signature $G T S i g=\langle G S, C, P, Q\rangle$ is a five-tuple GTSpec= $\langle\mathcal{V}, \mathcal{P} \mathcal{D}, F C, I, F\rangle$ where $\mathcal{V}: \mathcal{S F}_{Q}(C) \rightarrow \mathcal{P}\left(S_{C}\right)$ is a valuation of state formulas, and

$-\mathcal{P D}$ is a set of production definitions of the form $p(a): s p$ if $A C$, where $s p=(L \longleftarrow K \longrightarrow R)$ is a production span, $p(a) \in \mathcal{T} \mathcal{E}_{P}(L)$ is a transition expression over $L$, and $A C \subseteq \mathcal{S F}_{Q}(L)$ is a set of state formulas over $L$ representing application conditions.

- $F C=\langle I S, O S\rangle$ is a frame condition

$-I \subseteq S_{C}$ is a set of initial states

$-F=\langle$ weak, strong $\rangle$ is a fairness condition ${ }^{7}$ where weak, strong $\subseteq \mathcal{T} \mathcal{E}_{P}(C)$

${ }^{6}$ Here, the reduct is extended from graphs and graph morphisms to diagrams in $\mathcal{G} r(G S)$. This is possible because it forms a functor, called forgetful functor in [EM85]. It follows from the component-wise construction of pullbacks and pushouts in $\mathcal{G} r(G S)$ that their reduct is a pullback or pushout again. Hence, it preserves direct derivations as well as transitions.

${ }^{7}$ In [MP92] weak and strong fairness are called justice and fairness, respectively. 
Example 15. The graph transition specification MUTEX $=\left\langle\mathcal{V}_{\text {MUTEX }}\right.$, $\left.\mathcal{P} \mathcal{D}_{S Y S} \cup \mathcal{P} \mathcal{D}_{T R} \cup \mathcal{P} \mathcal{D}_{D D D}, F C_{M U T E X}, I_{M U T E X}, F_{M U T E X}\right\rangle$ is given by

- the obvious valuation $\mathcal{V}_{M U T E X}$ of state formula symbols, e.g., $g: G \rightarrow C \in$ $\mathcal{V}_{M U T E X}$ (no_request $(p, r)$ ) iff there is no request edge from $p$ to $r$ in $g(G)$.

- $\mathcal{P} \mathcal{D}_{S Y S}, \mathcal{P} \mathcal{D}_{T R}$, and $\mathcal{P} \mathcal{D}_{D D D}$ are given, respectively, by the productions in the upper, middle and lower part of Figure 2. The second TR-production, e.g., leads to the definition $r e q(p, r)$ : $s p_{r e q}$ if $\{$ no_request $(p)$, not_held_by $(r, p)\}$.

- FC MUTEX $=\langle\emptyset, \emptyset\rangle$ that is, the specification is considered as complete.

- IMUTEX is the set of all states $g: G_{0} \rightarrow C$ where $G_{0}$ is the graph in the left of Figure 3, which is put in all possible naming contexts.

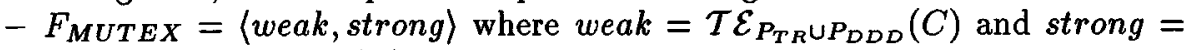
$\mathcal{T} \mathcal{E}_{\{\text {rel:PR, unlock:PR\} }}(C)$

A transition expression $p(c) \in \mathcal{T} \mathcal{E}_{P}(C)$ is enabled by a state $g$ if there is a transition $g \stackrel{L}{\sim} h$ with $p(c) \in L$. Then, a run $\rho: g_{0} g_{1} g_{2} \ldots$ satisfies the fairness condition $F=\langle$ weak, strong $\rangle$ if

Weak Fairness: For all $p(c) \in$ weak and $k \in \mathbb{N}$ : if for an $i \geq k, g_{i}$ enables $p(c)$, then there exists $n \geq k$ such that $g_{n} \stackrel{L}{\sim} g_{n+1}$ with $p(c) \in L$.

Strong Fairness: For all $p(c) \in$ strong and $k \in \mathbb{N}$ : if there exist infinitely many $i \geq k$ where $g_{i}$ enables $p(c)$, then there exist infinitely many $n \geq k$ such that $g_{n} \stackrel{L}{\sim} g_{n+1}$ with $p(c) \in L$.

Hence, the weak fairness condition of $F_{M U T E X}$ above says that a TR- or DDDtransition which is continuously enabled must eventually take place. The strong fairness condition implies that transitions using rel $: P R$ or unlock $: P R$ which are enabled infinitely many times, must be taken infinitely often.

Each graph transition specification generates a transition systems:

Definition 16 (generated transition system, correctness). The transition specification $G T$ Spec $=\langle\mathcal{V}, \mathcal{P D}, F C, I, F\rangle$ over GTSig generates the transition system $T S(G T S p e c)=\langle\mathcal{V}, \sim, \mathcal{R}\rangle$ where

- $g \stackrel{L}{\sim} h$ holds for two states $g: G \rightarrow C$ and $h: H \rightarrow C$ iff there exists a parallel graph transition $G \stackrel{d}{\stackrel{d}{\sim}} H$ respecting $F C$ with

- production span $\mathcal{P} a r(S p(L))$ where

$$
S p(L)=\{s p \mid p(c) \in L \wedge p(a): s p \text { if } A C \in \mathcal{P D}\}
$$

is the set of all production spans for production names in $L$

- bottom span $t=(G \longleftarrow D \longrightarrow H)$ given by the pullback of $g$ and $h$ modeling the "intersection" $D$ of $G$ and $H$ in $C$,

such that $c=g \circ m \circ i n_{s p}(a)^{8}$ for all $p(c) \in L$ with $p(a): s p$ if $A C$, i.e., the formal parameter $a$ is mapped to the actual parameter $c$, and the application conditions are satisfied, that is, $g \in \mathcal{V}\left(q\left(g \circ m \circ i n_{s p}(b)\right)\right)$ for all $q(b) \in A C$.

${ }^{8}$ Here, $m$ is the match of the DPB diagram $d$, and $i n_{s p}$ the embedding of the left-hand side of $s p$ in the parallel production span. 
- each run $\rho: g_{0} g_{1} g_{2} \ldots$ in $\mathcal{R}$ starts in an initial state $\left(g_{0} \in I\right)$ and satisfies the fairness conditions $F$.

The transition specification GTSpec is correct w.r.t. $X: \Phi$ iff $\models_{T S(G T S p e c)} X: \Phi$.

\section{Compositional Verification of System Properties}

This section formalizes the concept of a view on a specification. The idea is to ensure that the behavior of the overall specification is permitted by the view's loose semantics.

Definition 17 (view on a specification). Let GTSig $=\langle G S, C, P, Q\rangle$ and GTSig $=\left\langle G S, C, P_{0}, Q_{0}\right\rangle$ be graph transition signatures such that $P_{0} \subseteq$ $P$ and $Q_{0} \subseteq Q$. Then, a graph transition specification GTSpec 0 $\left\langle\mathcal{V}_{0}, \mathcal{P} \mathcal{D}_{0}, F C_{0}, I_{0}, F_{0}\right\rangle$ over $G T S i g_{0}$ is a view of GTSpec $=\langle\mathcal{V}, \mathcal{P D}, F C, I, F\rangle$ over GTSig, written GTSpec 0 GTSpec, iff $\mathcal{V}_{0}=\left.\mathcal{V}\right|_{\mathcal{S F}_{Q_{0}}(C)}, \mathcal{P} \mathcal{D}_{0} \subseteq$ $\mathcal{P D}, F C \subseteq F C_{0}, I \subseteq I_{0}$ and $F_{0} \subseteq F$, such that for each hidden production name $p \in P \backslash P_{0}$ with definition $p(a):(L \stackrel{l}{\longleftarrow} K \stackrel{r}{\longrightarrow} R)$ if $A C$, the reduct of $l$ and $r$ to non-input and non-output sorts, $(l)_{\overline{I S_{0}}}$ and $(r)_{\overline{O S_{0}}}$, respectively, yields isomorphisms.

Both transition specifications have the same graph signature $G S$ and context graph $C$, that is, their generated transition systems have the same set of states. All input (output) sorts in GTSpec are also input (output) sorts in the view. Hence, the frame conditions in GTSpec are stronger, which means that GTSpec is the more complete specification and effects that are covered by the loose semantics of GTSpec 0 are explicitly specified in GTSpec. On the other hand, each production of GTSpec which is hidden from the view must not have effects on its private sorts since for those the view is assumed to be complete.

A view of GTSpec can be induced by choosing a suitable subset of production names $P_{0}$ together with a frame condition $F C_{0}$. This choice is valid if the five-tuple GTSpec $c_{0}=\left\langle\left.\mathcal{V}\right|_{\mathcal{S F}_{Q_{0}}(C)}, \mathcal{P} \mathcal{D}_{0}, F C_{0}, I, F_{0}\right\rangle$ where $\mathcal{P} \mathcal{D}_{0}=\{p(a)$ : $s p$ if $\left.A C \in \mathcal{P D} \mid p \in P_{0}\right\}$ and $F_{0}=\left\langle\right.$ weak $\cap \mathcal{T} \mathcal{E}_{P_{0}}(C)$, strong $\left.\cap \mathcal{T E}_{P_{0}}(C)\right\rangle$ for $F=\langle$ weak, strong $\rangle$ is indeed a view of GTSpec.

Example 18. The DDD-view of the MUTEX specification is induced by the DDD-productions with the private sort blocked. The TR-view is given by all TRproductions and the frame condition $I S=\{P, R$, next, token, blocked, release $\}$ and $O S=I S \backslash\{$ release $\}$.

Semantically, GTSpec ${ }_{0} \subseteq G T S p e c$ implies that every run in GTSpec is also a run in $G T S$ Spec $_{0}$, or vice versa, the extension of a specification leads to a restricted behavior.

Proposition 19 (restriction of behavior). Let GTSpec 0 and GTSpec be transition specifications such that GTSpec $c_{0} \subseteq$ GTSpec, TS(GTSpec) $=$ $\langle\mathcal{V}, \leadsto, \mathcal{R}\rangle$, and $T S\left(G T S p e c_{0}\right)=\left\langle\mathcal{V}_{0}, \sim \sim_{0}, \mathcal{R}_{0}\right\rangle$. Then, $\mathcal{R} \subseteq \mathcal{R}_{0}$. 
Proof. A sequence of states $\rho$ is a run in GTSpec iff each pair of consecutive states $g, h$ is related by $g \stackrel{L}{\sim} h$ (i), $\rho$ starts in an initial state (ii), and it satisfies the fairness conditions (iii). In this case, $\rho$ is also a run in GTSpec 0 : For (i), $g \stackrel{L}{\sim} h$ implies $g \stackrel{L^{\prime}}{\sim} h$ where $L^{\prime}=\left\{p(c) \in L \mid p \in P_{0}\right\}$, because $\mathcal{P} \mathcal{D}_{0} \subseteq \mathcal{P D}$. If (the parallel graph transition generating) $g \stackrel{L}{\sim} h$ respects $F C$, then it also respects the weaker condition $F C_{0}$. In this case, also $g \stackrel{L^{\prime}}{\rightarrow_{0}} h$ respects $F C_{0}$ since the hidden productions in $P \backslash P_{0}$ do not create anything of non-input sort or delete anything of non-output sort, which concludes the proof of (i).

By assumption, $I \subseteq I_{0}$, that is if $\rho(0) \in I$ then $\rho(0) \in I_{0}$ implying (ii). For (iii), $F_{0} \subseteq F$ that is, the fairness condition is weakened. A state $g$ enables a transition expression $p(c)$ in $T S\left(G T S p e c_{0}\right)$ if and only if it enables $p(c)$ in $T S(G T S p e c)$. This follows from the fact that, on the one hand, $g \stackrel{L}{\sim} h$ implies $g \stackrel{L^{\prime}}{\sim} h$, as shown above. On the other hand, $g \stackrel{L^{\prime}}{\sim} h$ implies $g \stackrel{L}{\sim} k$, for some state $k \in S_{C}$ because, by default, all transitions are safe, and strengthening the frame conditions does not disable safe transitions.

This result is based on the notion of (safe) graph transition which anticipates the additional effects of the rules in the bigger specification. The main theoretical result of this paper follows from the definition of correctness and the proposition above.

Theorem 20 (correctness of compositional verification). Let GTSpec 0 and GTSpec be graph transition specifications such that GTSpec $c_{0} \subseteq$ GTSpec, and $X: \Phi \in \mathcal{T} \mathcal{F}_{G T S i g_{0}}$ be a temporal formula such that GTSpec 0 is correct w.r.t. $X: \Phi$. Then, also GTSpec is correct w.r.t. $X: \Phi$.

This allows to verify the properties of the MUTEX specification in a compositional way, by analyzing its views separately and deriving the MUTEX properties from the properties the views:

Example 21. For safety properties, the decomposition of the specification depends on the property to be shown. In order to prove MUTEX-safe, the stronger consistency condition $\square M U T E X-c c$ is established, where MUTEX-cc is satisfied in a state $g: G \rightarrow C$ iff for each resource $r \in g(G)$ there is a unique token edge $t$ with source $(t)=r$, or a unique held_by edge $h$ with source $(h)=r$, or a unique release edge $e$ with source $(e)=r$. This property is verified for the view induced by the production names $\{$ mount, unmount : $R$,pass : $P R$, take : PR, rel : $P R$, give $: P R$, unlock $: P R\}$ with private sorts $\{R$, held_by,token, release $\}$, i.e., all other sorts are input and output sorts. This is obviously a view of MUTEX since all the productions not mentioned do not affect the private sorts. Moreover, it is correct w.r.t. MUTEX-cc. In fact, the initial graphs satisfy the constraint and, since the relevant sorts are private and the condition is not violated by the productions of the view, the constraint is preserved by all transitions. By Theorem 20 one concludes that the complete specification MUTEX is correct w.r.t. $M U T E X-c c$ and hence w.r.t. MUTEX-safe. 
Another basic consistency condition is the integrity of the ring structure. Here, ring-cc shall be satisfied by a state $g$ if its next edges form a cycle containing all processes of the state. For proving $\square$ ring-cc let's pick up the view given by $P_{0}=\{n e w, k i l l: P\}$ with private sorts $\{P, n e x t\}$. Again, the chosen productions are the only ones affecting the private sorts, and it is similarly easy to see that the view is correct. Hence, also MUTEX is correct w.r.t. $\square$ ring-cc.

It is worth mentioning here that most of the requirements checked so far can be verified automatically: Given the production names and frame conditions, it is ease to test if they induce a valid view, and for verifying that the productions preserve certain consistency conditions, a procedure has been developed in [HW95].

The more difficult task is presented by the verification of liveness properties. Here, MUTEX-live has been decomposed into the local properties DDD-live and $T R$-live. Since the focus is on the composition of properties rather than on their verification for views, I do not present a proof of the correctness of $D D D$ and $T R$. Notice, however, that the consistency properties above have to be assumed.

Like safety properties, also the liveness properties $D D D$-live and $T R$-live carry over to the global system by Theorem 20. Combining them one obtains abounded $(n) \Longrightarrow$ MUTEX-live, that is, the boundedness of the system is left as an assumption for the schedule of SYS-productions.

\section{Conclusion}

In this paper I introduced graph transitions as a loose semantics of productions. By formalizing the notion of view I provided conceptual tools for compositional verification, and proved their correctness. An example showed the usefulness of the approach for specifying reactive systems and verifying their properties.

This notion of view is not very flexible yet since it does not allow the renaming of types or the extension of productions. It is not difficult, however, to generalize the concepts and results to some sort of specification morphisms, like the view relations in [EHTE97] or GTS morphisms in [HCEL96]. These papers also consider the problem of composing specifications.

A topic of ongoing work is the verification of views w.r.t. temporal properties. First results have been presented in [GHK] using graphical constraints [HW95] in order to define the evaluation of state formulas.

Acknowledgments Thanks to Martin Große-Rhode for helpful discussions and careful reading of the draft, and to Manuel Koch and Fabio Gadducci for joint work on temporal logic for graph transformation.

\section{References}

[CMH83] K.M. Chandy, J. Misra, and L.M. Haas. Distributed deadlock detection. ACM Transactions on Computer Systems, 1:144-156, May 1983. 
[CMR ${ }^{+}$97] A. Corradini, U. Montanari, F. Rossi, H. Ehrig, R. Heckel, and M. Löwe. Algebraic approaches to graph transformation I: Basic concepts and double pushout approach. In G. Rozenberg, editor, Handbook of Graph Grammars and Computing by Graph transformation, Volume 1: Foundations. World Scientific, 1997.

[EHTE97] G. Engels, R. Heckel, G. Taentzer, and H. Ehrig. A view-oriented approach to system modelling based on graph transformation. In Proc. of ESEC/FSE'97, Zürich, Springer LNCS 1301, 1997. Extended version to appear in International Journal of Software Engineering and Knowledge Engineering, 1998.

[EM85] H. Ehrig and B. Mahr. Fundamentals of Algebraic Specification 1: Equations and Initial Semantics, volume 6 of EATCS Monographs on Theoretical Computer Science. Springer Verlag, Berlin, 1985.

[EPS73] H. Ehrig, M. Pfender, and H.J. Schneider. Graph grammars: an algebraic approach. In 14th Annual IEEE Symposium on Switching and Automata Theory, pages 167-180. IEEE, 1973.

$\left[\mathrm{FKN}^{+92}\right.$ A. Finkelstein, J. Kramer, B. Nuseibeh, M. Goedicke, and L. Finkelstein. Viewpoints: A framework for integrating multiple perspectives in system development. International Journal of Software Engineering and Knowledge Engineering, 2(1):31-58, March 1992.

[GHK] F. Gadducci, R. Heckel, and M. Koch. Combining graph transformations with temporal logic. In First TMR GETGRATS Workshop, Bordeaux, October 1997.

[GR97] M. Große-Rhode. Algebra transformation systems and their composition. In this volume.

[HCEL96] R. Heckel, A. Corradini, H. Ehrig, and M. Löwe. Horizontal and vertical structuring of typed graph transformation systems. Math. Struc. in Comp. Science, 6(6):613-648, 1996. Also Techn. Rep. 96-22, TU Berlin.

[HEWC97a] R. Heckel, H. Ehrig, U. Wolter, and A. Corradini. Integrating the specification techniques of graph transformation and temporal logic. In Proc. of MFCS'97, Bratislava, Springer LNCS 1295, 1997.

[HEWC97b] R. Heckel, H. Ehrig, U. Wolter, and A. Corradini. Loose semantics and constraints for graph transformation systems. Techn. Rep. 97-07, TU Berlin, 1997. http://www.cs.tu-berlin.de/cs/ifb/TechnBerichteListe.html.

[HHT96] A. Habel, R. Heckel, and G. Taentzer. Graph grammars with negative application conditions. Fundamenta Informaticae, 26(3,4), 1996.

[HW95] R. Heckel and A. Wagner. Ensuring consistency of conditional graph grammars - a constructive approach. Proc. of SEGRAGRA'95 "Graph Rewriting and Computation", Electronic Notes of TCS, 2, 1995. http://www.elsevier.nl/locate/entcs/volume2.html.

[Löw93] M. Löwe. Algebraic approach to single-pushout graph transformation. TCS, 109:181-224, 1993.

[MP92] Zohar Manna and Amir Pnueli. The Temporal Logic of Reactive and Concurrent Systems, Specification. Springer-Verlag, 1992.

[Sch94] Enno Scholz. Verteilte Betriebssysteme. Seminar notes at the graduate college "Communication-based Systems", TU Berlin, 1994. 